\title{
The use of matrix manipulation programs in teaching statistical methods
}

\author{
N. JOHN CASTELLAN, JR. \\ Indiana University, Bloomington, Indiana 47405
}

\begin{abstract}
An array manipulation package, the Symbolic Matrix Interpretive System and Statistical Interpretive System (SMIS/SIS), is described. Using SMIS/SIS, one can declare matrices and arrays of real or randomly generated data and then manipulate the matrices using simple commands to produce virtually any desired analysis. The package, designed for use by nonprogrammers and programmers alike, features a full complement of matrix algebra operations and a smaller set of statistical functions. The package, while useful as a research tool, is especially useful in teaching statistical methods, particularly multivariate analysis in which the correspondence between mathematical formulas and program code has definite pedagogical advantages over the use of statistical program packages like SPSS, BMDP, and SAS.
\end{abstract}

The widespread availability of sophisticated programs for statistical analysis (BMDP, SPSS, SAS, etc.) makes it possible for anyone with access to a computer or terminal to perform very complicated analyses of data with a minimum of effort or understanding. This, coupled with the relative complexity of the mathematical and statistical bases for many of the procedures, poses special challenges to teachers of statistical methods, particularly multivariate techniques.

In order to avoid confronting mathematical complexity, some instructors have retreated to superficial treatment of the procedures and have relied on the statistical program packages or user manuals to make pedagogical points. Others have directly confronted the mathematical problems and, much to the chagrin of their students, have gone through derivations and procedures that seem remote from the neat summary analyses found in the application packages. In a recent paper, Thisted (1979) discussed the teaching of statistical computing courses that make extensive use of statistical program packages like SPSS, SAS, and BMDP. $\mathrm{He}$ (and several discussants in the same issue of the journal) outlined the advantages and disadvantages of such a course and provided useful caveats based on classroom experience and clear statistical pedagogical principles.

A different approach is to utilize special computer programs that enable the student to become facile with the mathematical techniques and to perform the statistical analyses directly relevant to the techniques and procedures being studied. One computer package that facilitates this is the Symbolic Matrix Interpretive System and Statistical Interpretive System (SMIS/SIS). In SMIS/SIS one can declare matrices and arrays of real or randomly generated data very easily and then manipu-

Requests for reprints should be sent to N. John Castellan, Jr., Department of Psychology, Indiana University, Bloomington, Indiana 47405. late them to produce any desired analysis.

The advantage of a system like SMIS/SIS is that it requires no prior knowledge of programming, the operations on matrices are easily stated or specified, and, when applied to techniques like multivariate analysis, provide a close link between mathematical formulation and final analysis. Indeed, the user has such control over computations that assumptions that often are buried deep within a package like SPSS become completely evident to the user.

\section{BACKGROUND}

During the past 15 years or so, a large number of program packages have become available for use in the statistical analysis of data. Among the first was the BMD statistical package developed at UCLA. This was followed by dozens of other packages of varying utility to researchers desiring to analyze anything from simple univariate data sets to complex surveys. The extent and diversity of such packages has been chronicled in at least three reviews (Anderson \& Coover, 1972; Berk, 1977; Schucany, Minton, \& Shannon, 1972).

Unfortunately, many packages that are especially well suited to the analysis of data are pedagogical disasters when it comes to use in the teaching of statistical techniques. On the other hand, some computerbased instructional materials are especially useful in the teaching of statistical techniques. However, many of these latter techniques either have little usefulness outside the classroom and instructional laboratory or are not designed for the routine analysis of experimental data. Examples are found readily in the "Proceedings" volumes of the Annual Conferences on Computers in the Undergraduate Curricula.

Over the last several years, I have been engaged in the development of computer-based data-analytic materials that are both useful in the classroom training situation and can be used in the analysis of relatively 
complex data sets found outside the classroom. ${ }^{1}$ The design of these materials has been based on the following set of criteria. (1) The associated "language" should be easy to learn and use. (2) The coded language should execute fairly rapidly. However, execution should include programming time and "compilation" time, if any. (3) The language should be modular in order that functions may be added with relative ease. (4) The language should be simple so that routine calculations and analyses are straightforward. However, it should be general enough that sophisticated and powerful programs may be written and the user not constrained by preconceived parameter restrictions or other arbitrary limits on the computational process. (5) The language should be generalizable to several different computer systems, since in the academic environment there is a great deal of sharing of programs. Consequently, transportability is a goal.

On the surface it might appear that I am describing desirable characteristics of a language like FORTRAN, PASCAL, or BASIC. However, there are many difficulties and weaknesses inherent in such languagesespecially for the neophyte programmer. The problems that are particularly critical are the input-output conventions and procedures for the handling of arrays and subscripts. As many instructors can attest, the majority of errors made by students concern input-output, subscripts, array manipulations, and so on. While some of these difficulties are minimized in some extended versions of FORTRAN and BASIC, they are not eliminated. The use of the various "scientific subroutine" packages is also helpful, but it does not eliminate all of the problems-indeed, it creates others as well.

Another problem is that of debugging. In a typical compiled program, one cannot assess the validity of the coding until all compilation errors are removed; such a debugging procedure often is inefficient but is a necessary feature of the language. Finally, and most important, we wanted a language that could be learned easily but still had considerable power, for we are attempting to train people in statistical techniques, not computer programming.

After evaluation of several packages then available, we turned to an interpretive language design originally developed at the University of California for use in the solution of engineering problems. After some modification, enhancement, and generalization, SMIS/SIS emerged (Castellan, 1970, 1974). The system is available and in use at several colleges and universities as well as at some research institutes.

The primary intent of this system was to provide a simple programming system that could be used to solve rather complicated problems. The system itself is not really complete and is not designed to compete with such extensive analysis systems as SPSS. It is a simple system. At the same time, it is more powerful and more easily programmed than other array manipulation systems like OMNITAB. The major development effort has been directed toward the use of the matrix interpre- tive system (SMIS) for power, and the statistical interpretive system (SIS) for the analysis of simple statistical problems. Both are used for pedagogical purposes in the classroom.

\section{BRIEF OVERVIEW OF SMIS/SIS}

The basic design of the system is quite simple, and students often can be taught to program the language in less time than it takes to learn to use a keypunch or terminal. The following is a brief overview of the structure of SMIS/SIS. There are three types of input records in the system: operation, data, and remark cards. Although it is easy to implement SMIS/SIS from an interactive terminal, for convenience and clarity, the following discussion will refer to input records as cards.

Remark cards are simply comments the programmer adds to the program to make it more readable or to provide special labels, and so on.

Data cards are records that contain input data and arrays. The basic unit of data in SMIS/SIS is an array or matrix. Once an array has been defined and assigned an alphanumeric name, all subsequent references to the matrix are by name only. Matrices derived from operations (such as matrix addition, computation of correlation matrices, etc.) have their dimensionality automatically generated. Thus a series of operations on sets of matrices with differing initial dimensions would vary only at the point where the original matrices were input or defined.

Operation cards are the records that control the flow of the program. Each operation card consists of an operation name and from 0 to 10 parameters, depending upon the specific operation to be performed. The parameters are the names of matrices to be manipulated and scalars and indices necessary to specify the options to be taken in the particular operation. At the present time there are 48 matrix manipulation operations and 23 statistical operations. These operations are summarized in the Appendix. In addition, the system is designed for easy access to user-written FORTRAN subroutines.

\section{Coding Examples}

Example 1. Suppose I wished to perform the simple task of adding two matrices and finding the inverse of the result.

$$
C=(A+B)^{-1}
$$

Here is how the SMIS coding would look.

$\begin{array}{llllll}\text { LOAD } & \text { A } & & & 5 & 5 \\ \quad \text { data } & \text { cards] } & & & & \\ \text { LOAD } & \text { B } & & & 5 & 5 \\ \quad \text { data } & \text { cards] } & & & & \\ \text { ADD } & \text { A } & \text { B } & \text { C } & & \\ \text { INVERT } & \text { C } & & & & \\ \text { PRINT } & \text { C } & & & \end{array}$


Note that the dimensionality of $\mathrm{A}$ and $\mathrm{B}$ is specified only in the LOAD instruction and that the matrix $\mathrm{C}$ is never overtly dimensioned.

Example 2. Suppose I wish to calculate some descriptive statistics for a set of data that consists of six variables for 50 subjects. I wish to calculate means, standard deviations, and product-moment correlations. The following is the complete SMIS/SIS code required.

LOAD DATA $(6 \mathrm{~F} 3.0) \quad 50 \quad 6$
$\quad \quad$ data cards]
CORRE DATA MEAN STD R
PRINT MEAN
PRINT STD
PRINT R

In this example the mnemonics should be selfexplanatory. MEAN and STD are 1 by 6 vectors, and R is a 6 by 6 matrix; they are created by the system when they are needed. The programmer could use any names at all for the arrays associated with the means, standard deviations, and correlations-The order is the critical factor. Also, the arrays MEAN, STD, and R may be used in later computations, if desired.

Suppose I also wanted to add the six scores for each of the 50 subjects, calculate various statistics for the derived total scores, and print a frequency distribution (or histogram). The coding inserted after the above would be (RSUM calculates a row sum):

\section{RSUM DATA TOTAL HISTO TOTAL}

Of course, one may continue with other operations as desired. In order to see how one might do some of the computations in this example using only the matrix routines, refer to Table 1 . Table 1 gives an example of computing a covariance matrix, correlation matrix, and performing a principal-components analysis using only matrix operations.

\section{VARIETY OF CLASSROOM USE AND SOME OUTCOMES}

SMIS/SIS has been used in two very different classroom situations, the diversity of which illustrates the simplicity and power of the system. It has been used in an undergraduate course in statistical techniques that has only high school mathematics as a prerequisite. The other course in which it has been used extensively is a graduate-level course in multivariate analysis.

In the undergraduate course, SMIS/SIS is used to solve simple computational problems, graph data, and do simple hypothesis testing. In addition, since SMIS/SIS

Table 1

Example of SMIS Coding to Produce Covariances, Correlations, and Principal Components

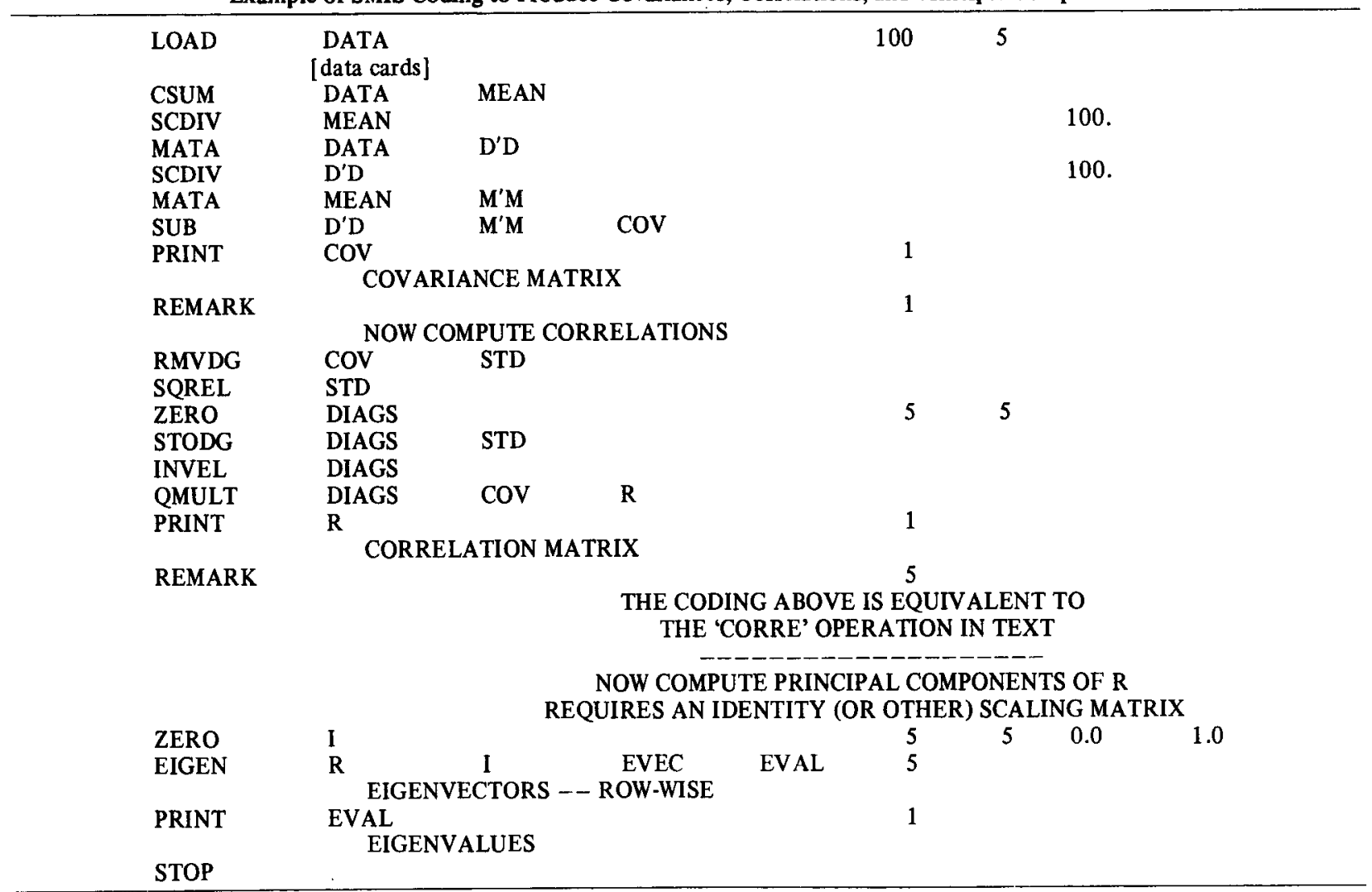


has the capability of generating random samples from different distributions, it is possible for the student to generate empirical sampling distributions and demonstrate limit theorems such as the central limit theorem. The student can "steer" the analysis, exercise judgment, and use the system in a constructive rather than cookbook fashion. Obviously, the system can be used with a wide variety of textbooks.

In the graduate course in multivariate analysis, the students use the statistical routines to some extent. However, all of the computational problems in the first 10 chapters of the popular textbook by Morrison (1976) can be programmed with relative ease by means of SMIS/SIS. The system has the advantage of letting the student program solutions to problems using matrix algebra, which is the notational base of the text.

How successful has SMIS/SIS been? At the undergraduate level, there have been some rather surprising results. Many of the students continue to use the system after completion of the course. While this might be considered natural for graduate students who could use the system in their research, we were surprised at the large number of undergraduates who used the system after completion of the statistics course to help friends in other courses to analyze data. (In fact, some enterprising students even set up a "consulting service" for students in an introductory laboratory course.) In performance on examinations in statistics, classes that have used SMIS/SIS have shown a greater understanding of statistics in general.

Several persons have used SMIS/SIS to debug and validate other programs. For example, at the University of Colorado, SMIS/SIS was used to check out a general multivariate analysis program that was being developed. The advantage of SMIS/SIS was that the sequences of operations are clear, precise, and unambiguous with respect to intended outcome.

In conclusion, SMIS/SIS, which is useful in general analysis situations, has been well adapted to classroom use, for not only does it help students understand statistical concepts, it has been used spontaneously in other contexts at a later time.

\section{REFERENCES}

ANDERSON, R. E. Instruction in social data analysis with the MISSIS system. Behavior Research Methods \& Instrumentation, 1980, 12, 165-171.

Anderson, R. E., \& Coover, E. R. Wrapping up the package: Some thoughts on applications software for social data analysis. Computers and the Humanities, 1972, 7, 81-85.

BЕRк. R. A. Survey of integrated statistical computer packages. Behavior Research Methods \& Instrumentation, 1977, 9, 270-280.

Castellan, N. J., JR. SMIS/SIS: The symbolic matrix interpretive system and the statistical interpretive system. COMMON: Seattle Proceedings, 1970, 64-73.

Castellan, N. J., JR. On the instructional use of SMIS/SIS (Symbolic Matrix Interpretive System). In J. L. Gillings \& J. A. Barney (Eds.), Proceedings of the First Indiana University Computer Network Conference on Computer Related Curriculum Materials, 1974, 162-169.

Morrison, D. F. Multivariate statistical methods (2nd ed.). New York: McGraw-Hill, 1976.

Schucany, W. R., Minton, P. D., \& Shannon, B. S., Jr. A survey of statistical packages. Computing Surveys, 1972, 4, 65-79.

Thisted, R. A. Teaching statistical computing using computer packages. American Statistician, 1979, 33, 27-35.

\section{NOTE}

1. Anderson (1980) describes the Minnesota Interactive Statistical System for Instruction in Sociology (MISSIS), which arose out of similar concerns and shares many design principles with SMIS/SIS.

\section{APPENDIX}

SMIS: Symbolic Matrix Interpretive System Operations

START
STOP
REMARK
LOAD
PRINT
DELETE
PUNCH
OUTAPE
WRITE
READ
REWIND
BACKSP

DUPL
ZERO
TRANS
STODG
RMVDG
STOSM
RMVSM

START

STOP

REMARK

LOAD

PUINT

PUNCH

OUTAPE

REWIND

BACKSP

DUPL

TRANS

RMVSM
Delete all matrices from storage, begin new problem.

End all operations.

Print comments.

Input/Output

Load (read) matrix from cards or input file.

Print a matrix.

Delete a matrix from storage.

Punch a matrix.

Write a matrix onto tape or file (BCD).

Write a matrix onto tape or file (binary).

Read a matrix from tape or file (binary).

Rewind tape.

Backspace tape.

Matrix Building

Duplicate a matrix.

Create a matrix with specified on-/off-diagonal elements.

Transpose a matrix.

Store a diagonal matrix in another matrix.

Remove diagonal from a matrix and store as a vector.

Store a submatrix in a larger matrix.

Remove a submatrix from a larger matrix. 


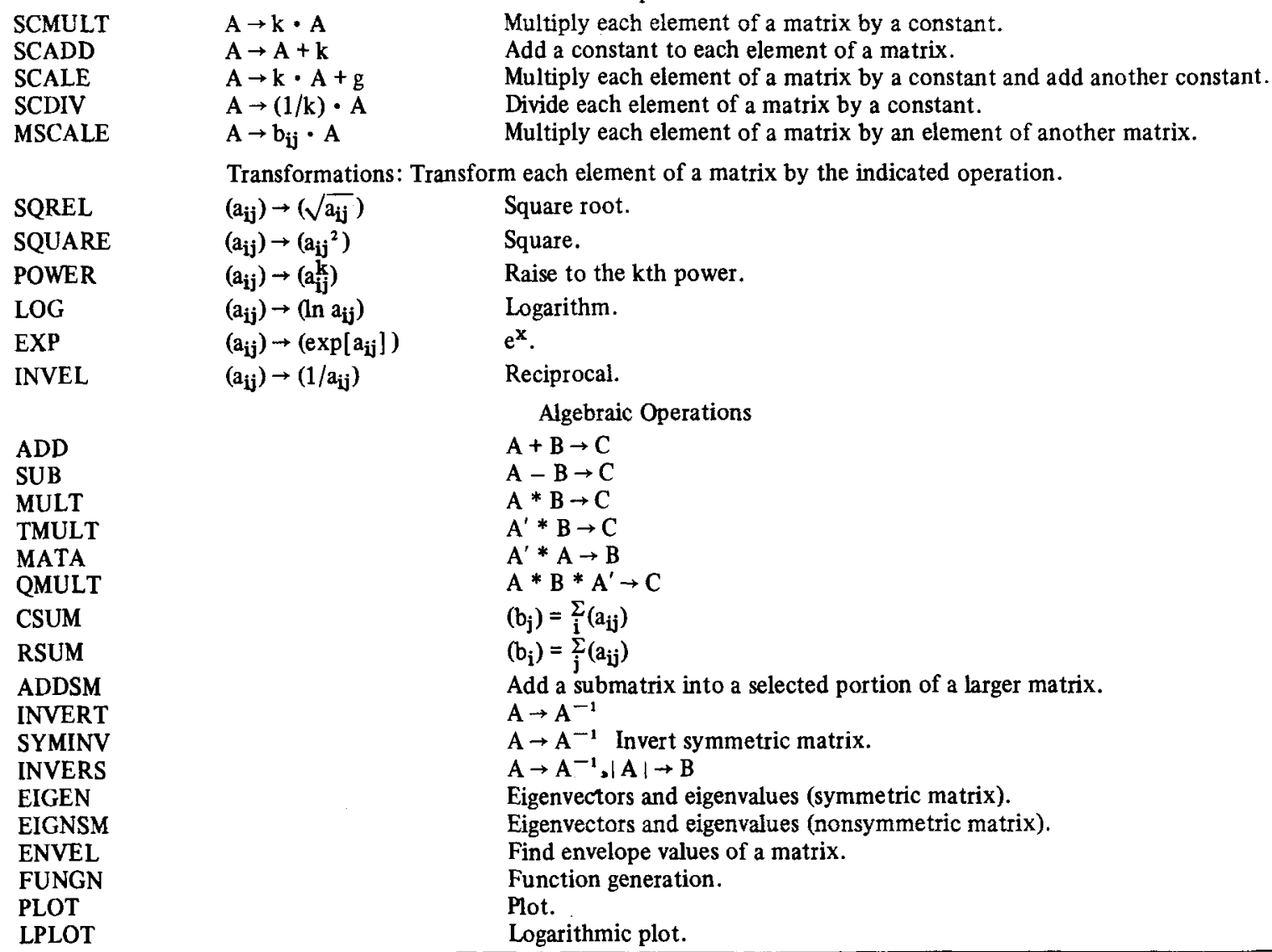

SIS: Statistical Interpretive System Operations*

TALLY

TAB1

TAB2

COVAR

MULTR

HISTO

CHISQ

X2FIT

TTEST

SRANK

KRANK

UTEST

WTEST
Descriptive Statistics

Calculate mean, standard deviation, minimum, and maximum for each variable in a data matrix.

Tabulate, for a selected variable in a data matrix, the frequencies or percent frequencies over class intervals. Also, for the same variable, calculate the total, average, standard deviation, minimum, and maximum.

Perform a two-way classification of the frequency or percent frequency and other statistics over given class intervals, for two selected variables in a data matrix.

Calculate means, standard deviations, and covariances for a data matrix. Perform multiple linear regression analysis for a selected dependent variable and a set of selected independent variables in a data matrix. Construct and print a histogram (frequency distribution).

\section{Statistical Tests}

For a contingency table, calculate chi square, degrees of freedom, and significance probability.

Perform chi-square goodness-of-fit test.

Calculate certain $t$ statistics on the means of populations under various hypotheses.

Calculate the Spearman rank correlation between two variables. Tied observations are assigned the average of the tied ranks.

Calculate the Kendall rank correlation coefficient for two variables.

Test whether two independent groups are from the same population by means of the Mann-Whitney U test.

Compute the Kendall coefficient of concordance for a given data matrix. 
CORRE Calculate means, standard deviations, and product-moment correlation coefficients for a data matrix.

FRIED Calculate the Friedman two-way analysis of variance statistic, given a data matrix.

QTEST Calculate the Cochran Q-test statistic, given a matrix of dichotomous data.

RANK

RANDOM

NORMAL

AUTO

CROSS

SMO
Miscellaneous

Assign rank-order numbers to the successive elements of the vector. Create a data matrix of uniformly distributed random numbers.

Create a data matrix of normally distributed random numbers.

Time-Series Analysis

Calculate the autocovariances for various lags for a time series of observations.

Calculate the crosscovariances of time series (B) lagging and leading time series (A) for various lags and leads.

Calculate the smoothed or filtered series (C), given a time series (A), a selection integer $\mathrm{N} 1$, and a weighting series (B).

EXSMO Calculate a smoothed series (B) given the time series (A) and the smoothing constant $\mathrm{S} 1$.

*Some of these operations may require a data matrix. A data matrix is a matrix in which the rows are observations or replications and the columns are assumed to be variables-either dependent or independent, or both. In many cases there may be only one variable (column) in the data matrix. Note that if a matrix is not in the above form, operation TRANS will make it so. Input vectors may be row or column vectors. All output vectors are row vectors unless noted otherwise. 